\title{
Derivation of Supersaturation and Nucleation Flux in a Stirred Tank from Tracer Concentration Measurements
}

\author{
By A. Fall, O. Lecoq*, and R. David
}

The PLIF (Planar Laser Induced Fluorescence Technique) method is applied to determine experimentally the field of supersaturation in a separate double jet reactive precipitator from the local instantaneous field of concentrations of an inert tracer. The nucleation flux in the mixing region is derived.

\section{Introduction}

The industrial production of solid particles by precipitation or reactive crystallization is usually carried out in mechanically stirred tanks. In these processes two or more liquid streams are brought into contact in a more or less controlled fashion. The contacting and mixing conditions of reactants govern the supersaturation distribution, consequently both nucleation and growth kinetics, which in turn affect the crystal size distribution, nature, morphology and purity. Such influences of mixing conditions on crystal products have been described in the literature [1].

Precipitation are generally characterized by small particles and short to medium precipitation times [2]. Houcine et al. [3] and Phillips et al. [4] have verified on different precipitates that the mixing geometries, which generate high variances of the concentration of an inert tracer during continuous double jet mixing with separate feed streams, correspond in a reactive semi-batch process with a single jet feeding (but located at one of the feed points and keeping the same geometry of the rest of the tank) to the production of smaller particle; and thus to higher overall nucleation rates.

The question of the relation between tracer experiments and extent of chemical reactions has been tackled by Levenspiel [5] several years ago. In between, Sheenoy and Toor [6] showed that there is a relation between feed fraction of an inert tracer and the extent of an instantaneous chemical reaction, provided that the molecules have similar diffusivities.

The aim of this paper is to apply laser sheet visualization and image processing technique to check if this type of correlation between variances and nucleation flux can be verified in the case of double jet operation of a precipitator with separated feed streams. We use fluorescent tracer solution and water as a double jet model mixing system in a stirred tank. In the first moments following the introduction of the fluid in the tank, the mixing of inert tracers with no production of solids is similar to the mixing of two reactants in ionic precipitation as there is no significant consumption of the reactants during the

[*] A. Fall (e-mail: Ahmed.Fall@enstimac.fr), O. Lecoq (corresponding author, e-mail: lecoq@enstimac.fr), R. David (e-mail: rdavid@enstimac.fr), Laboratoire de Génie des Procédés des Solides Divisés, UMR CNRS 2392, Ecole des Mines d'Albi-Carmaux, Campus Jarlard, 81013 ALBI CT cedex 09, France. first seconds of contact [7]. Thus, mixing in ionic precipitation can be characterized by following the mixing of two inert tracers, or mixing of a single tracer in solution with pure solvent, injected by two separate feed streams.

The Planar Laser Induced Fluorescence Technique (PLIF) combined with image processing which is a powerful method to characterize the mixing in stirred tanks is used, [8,9]. It consists of the measurement of the fluorescence intensity of a tracer excited by a very thin planar laser sheet. This intensity is transformed into a local instantaneous concentration field by a calibration procedure [8].

\section{Experimental Setup}

The simplified scheme of the apparatus used in this work (see [9]) is shown in Fig. 1. The experiments are carried out in a stirred tank of $20 \mathrm{dm}^{3}$ of standard configuration, with internal
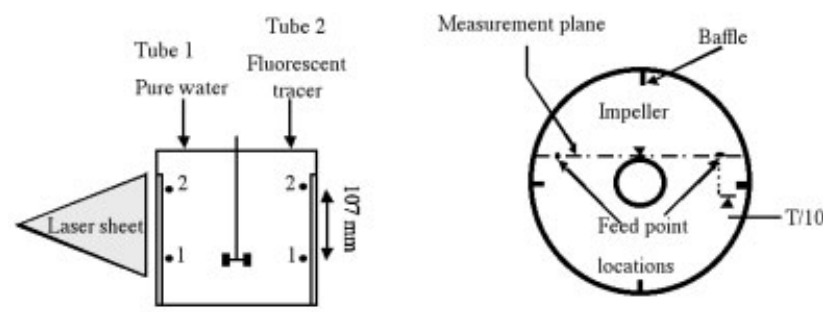

Figure 1. Experimental setup.

diameter $\mathrm{T}=0.29 \mathrm{~m}$. The walls of the inner tank are equipped with four baffles of width $\mathrm{T} / 10$ and thickness $\mathrm{T} / 100$ at intervals of $90^{\circ}$. The impeller diameter (Rushton turbine) and bottom clearance are equal to $\mathrm{H} / 3$, where $\mathrm{H}=$ Trepresents the height of the liquid in the $\operatorname{tank}^{1)}$.

The stirring speed is set at $\mathrm{N}=3.5 \mathrm{~s}^{-1}$. Two reservoirs continuously supply the tank with equal flow rates $\left(\mathrm{Q}_{\mathrm{A}}=\mathrm{Q}_{\mathrm{B}}\right.$ $=0.67 \mathrm{dm}^{3} \mathrm{~min}^{-1}$ ) by two incoming feed tubes (internal diameter $6 \mathrm{~mm}$ ). The two feed point locations are located at the tangential vertical plane to the impeller at $T / 10$ from the baffle; close to the radial discharge of the impeller (position 1 ), and close to the fluid surface (position 2). The tank is operated

1) List of symbols at the end of the paper. 
in the continuous mode for tracer concentration measurements. The tracer is Rhodamine B.

\section{Results and Discussion}

First, the local instantaneous field of concentrations is determined. From this, we deduce the mixing index, the average fraction coming from one of the feed streams, and the temporal variance. The field of supersaturation in the same tank when considering a double jet situation is calculated from our experimental data and from this field we deduce the nucleation flux in the mixing zones. The details of the equations used in the following section are available in [9].

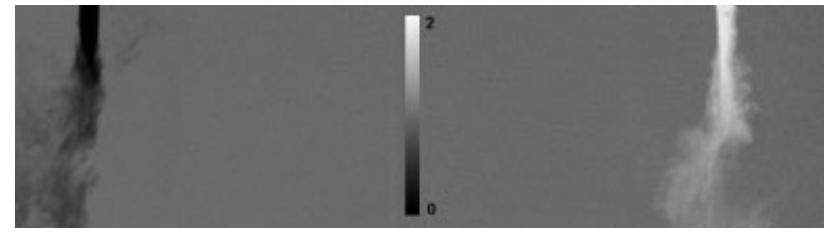

Figure 2. An example of the instantaneous reduced local concentration for position 2 .

An example of instantaneous reduced local concentration of the tracer is shown in Fig. 2 for the given geometry for feed streams location 2. It varies between 0 (black ) in the pure water feed stream to 2 (white) in the fluorescent tracer feed stream.

The instantaneous mixing index field is given by the equation below:

$\alpha(x, y, t)=1-\left|\frac{C_{T}(x, y, t)-C_{T 0}(x, y)}{(2 \times \bar{f}-1) C_{T}(x, y, t)+C_{T 0}(x, y)}\right|$

This index varies from 0 (black), in the feed stream jets corresponding to no contact between the two liquids up to a value close to 1 (white) in the rest of the tank. The average mixing index field is shown in Fig. 3, for the other feed streams location, i.e. position 1 .

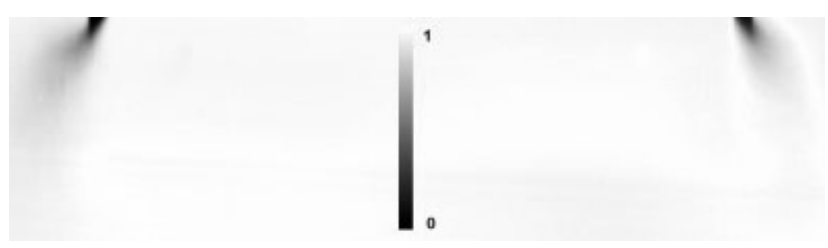

Figure 3. Average mixing index field for position 1.

As a consequence of symmetry, the results of the temporal variance, the supersaturation and the nucleation flux, are only given in one of the jets, i.e. the right one corresponding to the feed stream of the fluorescent tracer.

The temporal variance $\sigma^{2}(x, y, t)$ of local reduced concentration of the inert fluorescent tracer is deduced from the field of the concentration by Eq. (2): $\sigma^{2}(x, y, t)=\frac{1}{n} \sum_{n}\left(\frac{C_{T}(x, y, t)}{C_{m p}}-\frac{\bar{C}_{T}(x, y)}{C_{m p}}\right)^{2}$

Experimental convergence is observed with $\mathrm{n} \geq 100 . \sigma^{2}(x, y, t)$ quantifies the mixing dynamics in the injection zones (mixing zones). Houcine etal. [3] have shown that it is strongly correlated with the average crystal size produced in the feed jet region. Larger high variance zones of inert tracer concentration, during continuous mixing separate doublejet, correspond in reactive semi-batch single-jet to the production of smaller particles and vice versa.

Fig. 4 shows the field of the variance in the injection feed for two different feed point locations (see Fig. 1). When feeding
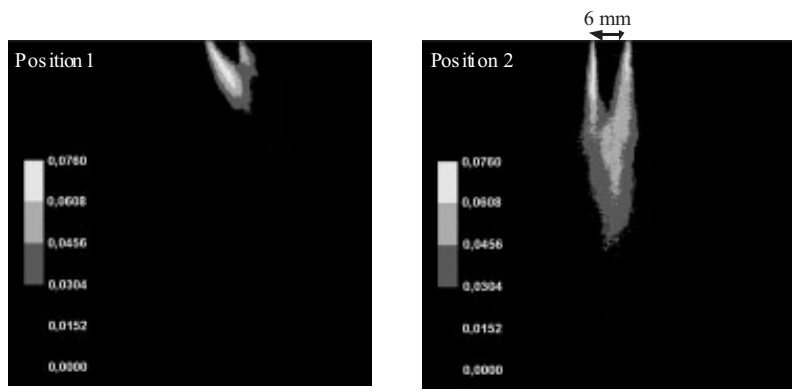

Figure 4. Variance field in the feed stream jet containing fluorescent tracer.

below the surface of the liquid (position 2) a larger high variance area (zone of non-zero variance) is obtained. Consequently, smaller crystals should be generated in position 2 compared to position 1 which is close to the impeller.

To go further in the analysis, it is interesting to calculate precipitation related variables such as supersaturation or nucleation flux. The local instantaneous supersaturation $S(x, y, t)$ resulting from two feed streams jets containing two reagents is derived from tracer concentrations [10]. It is assumed that a mixing region results from the mixture of surrounding fluid (composition $\bar{C}_{A}, \bar{C}_{B}$ ) and fresh entering fluid with concentrations $C_{A_{0}}^{\prime}$ and $C_{B_{0}}^{\prime}$ respectively. $S(x, y, t)$ represents the driving force for crystallization. When we pose $X_{T}=C_{T}(x, y, t) / C_{m p}, S(x, y, t)$ can be written:

$S(x, y, t) \approx \frac{\bar{C}_{A} C_{B_{0}}^{\prime}}{P_{S}}\left(X_{T}-X_{T}^{2}\right) \quad \begin{aligned} & \text { in the vicinity of the tube } 1 \\ & \text { (B-feed) }\end{aligned}$

$S(x, y, t) \approx \frac{C_{A_{0}}^{\prime} \bar{C}_{B}}{P_{S}}\left(3 X_{T}-X_{T}^{2}-2\right) \quad \begin{aligned} & \text { in the vicinity of the tube } 2 \\ & \text { (A-feed) }\end{aligned}$

The crystal properties are related to the supersaturation, which is directly influenced by the feed points location. Philips et al. [4] have observed that position 2 corresponds, in single jet reactive precipitation, to the production of smaller particles. This means that local supersaturations are higher in this case. Fig. 5 shows the field of time averaged supersaturation $\bar{S}(x, y, t) \times P_{S} /\left(C_{A_{0}}^{\prime} \bar{C}_{B}\right)=\bar{S}(x, y, t) / S_{0}^{\prime}$ calculated for the two 

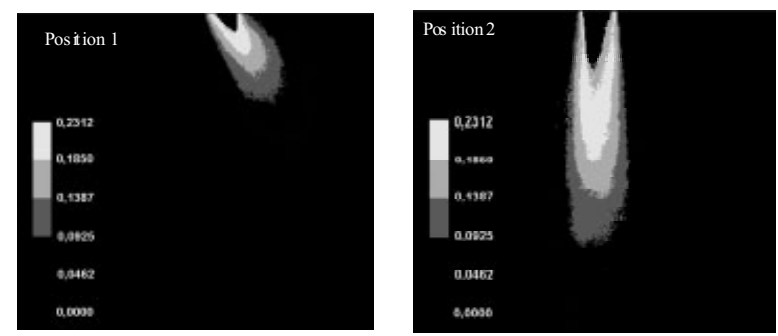

Figure 5. Reduced average supersaturation field in the feed stream containing fluorescent tracer.

positions. We can see that (a) the field is similar to the variance one (Fig. 4) with the same limits of the relevant area and (b) that position 2 induces the largest zones of both high supersaturation and variance, i.e. smaller particles, which is in agreement with the results of both Philips and Houcine.

The second crucial parameter to look for is the nucleation flux $\bar{R}_{N}$ in the mixing zones. According to the Volmer relation ([11]), it is given by the following equation:

$\bar{R}_{N} \propto \int_{X_{T}=0}^{X_{T}=2} \exp \left(\frac{-B_{N}}{\log _{e}^{2}(S(x, y, t))}\right) p\left(X_{T}\right) d X_{T}$

This parameter gives important information on the local time-averaged number of particles generated in the mixing zone, (Fig. 6). It is first noticeable that these fields are very similar to the previous supersaturation and variance fields
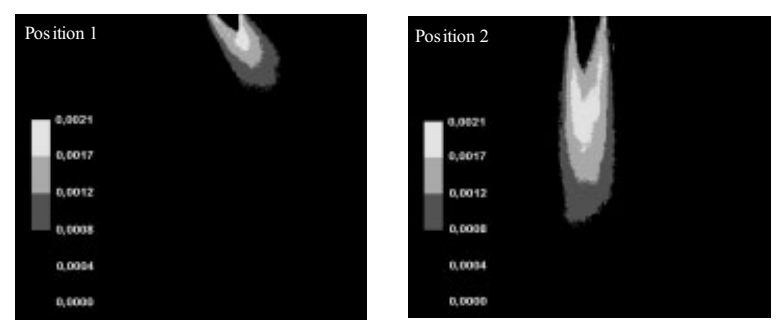

Figure 6. Nucleation-flux field in the feed stream jet containing fluorescent tracer (arbitrary $\bar{R}_{N}$ scale).

(Figs. 4 and 5). In the same way larger zones of nucleation flux are obtained for position 2 as compared to position 1. Again, feed position 2 should produce smaller crystals.

\section{Conclusions}

Application of the Planar Laser Induced Fluorescence Technique (PLIF), combined with image processing was used to predict qualitatively crystals size distribution in a standard precipitation tank. At one hand, we checked with respect to the literature results that the temporal variance field of tracer concentration with a separate double jet precipitator is correlated with the average nucleation rates. In the feed jet area, small regions of high variances correspond to larger crystals and vice versa. On the other hand, the fields of supersaturation in the same tank but in a reactive situation were calculated from our experimental data (the fields of the tracer concentration) and from this, we derived the nucleation flux in the mixing region. Results obtained with these two parameters are in accordance with those of Houcine and Phillips. This approach allows one to have a rapid check on the operating conditions to control both crystals size and properties in an industrial precipitator.

\section{Symbols used}

$\begin{array}{ll}B_{N} & {[-]} \\ C_{A} & {\left[\mathrm{~mol} \mathrm{~m}^{-3}\right]} \\ C_{A O}^{\prime} & {\left[\mathrm{mol} \mathrm{m}^{-3}\right]} \\ & \\ C_{B} & {\left[\mathrm{~mol} \mathrm{~m}^{-3}\right]} \\ C_{B O}^{\prime} & {\left[\mathrm{mol} \mathrm{m}^{-3}\right]} \\ & \\ C_{m p} & {\left[\mathrm{~mol} \mathrm{~m}^{-3}\right]} \\ C_{T} & {\left[\mathrm{~mol} \mathrm{~m}^{-3}\right]} \\ C_{T 0} & {\left[\mathrm{~mol} \mathrm{~m}^{-3}\right]}\end{array}$

constant, =100, Eq. (5)

concentration of fluorescent

tracer

feed concentration of

fluorescent tracer

concentration of pure water

feed concentration of pure

water

perfect mixing concentration

tracer local concentration

mixing tracer concentration in the outlet

$\bar{f}=Q_{B} /$

$\begin{array}{lll}\quad\left(Q_{A}+Q_{B}\right) & {[-]} & =0.5 \\ n & {[-]} & \text { number of images } \\ p\left(X_{T}\right) & {[-]} & \text { PDF of } \mathrm{X}_{\mathrm{T}} \text { at point }(\mathrm{x}, \mathrm{y}) \\ P_{S} & {\left[\mathrm{~mol}^{2} \mathrm{~m}^{-6}\right]} & \text { solubility product } \\ Q_{A}, Q_{B} & {[-]} & \text { feed rates } \\ R_{N} & {[-]} & \text { nucleation flux }\end{array}$

$\mathrm{S}_{0}^{\prime}=\mathrm{C}_{\mathrm{A}_{0}}^{\prime}$

$\begin{array}{ll}\quad \times \overline{\mathrm{C}_{\mathrm{B}}} / \mathrm{P}_{\mathrm{S}} & {[-]} \\ X_{T} & {[-]} \\ \sigma^{2} & {[-]} \\ \alpha_{A B} & {[-]} \\ - & {[-]}\end{array}$

$=1000$, Eq. $(4)$

reduced local concentration

reduced temporal variance

mixing index

time average

Tube 1 = water feed stream

Tube 2 = tracer feed stream

\section{References}

[1] R. David, Powder Technol. 2001, 121, 2.

[2] A. Mersmann, Crystallization Technology Handbook, Marcel Dekker Inc, New York 2001.

[3] I. Houcine, E. Plasari, R. David, J. Villermaux, Trans IChemE Part A 1997, 75, 252

[4] R. Phillips, S. Rohani, J. Baldyga, AIChE J. 1999, 45 (1), 82.

[5] O. Levenspiel, Chemical Reaction Engineering, Chapiter 10: Mixing of Fluids, Wiley \& Sons, New-York 1971.

[6] U. V. Sheenoy, H. L. Toor, AIChE J. 1990, 3 (2), 227.

[7] R. David, B. Marcant, AIChE J. 1994, 40 (3), 424

[8] I. Houcine, H. Vivier, E. Plasari, R. David, J. Villermaux, Experiment in Fluids 1996, 22, 95.

[9] A. Fall, O. Lecoq, R. David, Trans IChemE Part A 2001, 79, 876.

[10] R. David, A. Fall, O. Lecoq, Derivation of Supersaturation and Nucleation Flux during Precipitation from Mixing Pattern of an Inert Tracer in the Same Device: Case of Unmixed Feed Streams, Cem. Eng. Sci. 2002, acceüted.

[11] M. Volmer, A. Weber, Z. Phys. Chem. 1926, 119, 277. 